\title{
COMPARING THE RESISTANCE TO MOTION OF PISTONS COATED WITH A LAYER OF NANOTUBES WITH STANDARD PISTONS
}

\author{
Antoni Iskra, Maciej Babiak, Jarosław Kałużny \\ Poznan University of Technology \\ Institute of Combustion Engines and Transport \\ Piotrowo Street 3, 60-965 Poznan, Poland \\ tel.: +486166525 11, fax: +48616652204 \\ e-mail: antoni.iskra@put.poznan.pl \\ Michael Giersig \\ Freie Universitaet Berlin \\ Arnimallee 14, 14195 Berlin \\ tel.:00493083853047,fax:004983856299 \\ e-mail:giersieg@physik.fu-berlin.de \\ Krzysztof Kempa \\ Boston College \\ Chestnut Hill MA, 02467 Boston \\ tel.: +1 617552 3592, fax: +16175528478 \\ email:kempa@bc.edu
}

\begin{abstract}
The paper presents preliminary results of testing the resistance to motion of pistons coated with a layer of carbon nanotubes (CNTS). A significant part of this paper was devoted to the problems of putting a layer of nanotubes on the surface of an aluminum alloy. Obtaining a layer of nanotubes of a very narrow margin of tolerance was a difficult technological problem to overcome. A standard process of growing a layer of nanotubes leads to a corrosion damage of the side surface of pistons; therefore, new technologies were developed allowing for obtaining a permanent layer of nanotubes less than 5 microns thick. Pistons whose side surfaces were coated with a layer of nanotubes were mounted to an engine with an external drive, and then measurements of the moment of momentary resistance to motion were performed, which enables capturing these phases of the engine work cycles in which the layer of nanotubes gives the best results. At present, long-term research is being carried out in order to determine the degree of the risk of exfoliation of the layer of nanotubes under the conditions of large mechanical and thermal loads. The special nanotechnology method cold nanosphere lithography has been invested to control the structural properties sand growth of multiwalled carbon nanotubes. The preliminary analysis of dismantled pistons revealed that nanotubes layers were partially worn off at the peaks of micro roughness but in the valleys, the nanotubes accurately adhered to the piston lateral surface.
\end{abstract}

Keywords: friction in internal combustion engine, nanotubes layers

\section{Introduction}

Since the invention of the piston, combustion engine there has been research aimed at improving the parameters of work as well as durability of engine sets. In particular, the stability of surfaces of cooperating sets determines the durability of the engine. The most obvious sets, which are of our concern, are piston-cylinder groups, slide bearings of the crankshaft and timing gear systems. In principle, all surfaces moving against each other are enriched to some extent. Most 
frequently, the surface refinement is hardening or applying a layer of chromium or other materials ensuring good frictional properties. For the last several years, there has been a quick development of the possibilities of surface refinement by applying a layer of nanotubes.

Coating surfaces of alloys as well as ceramics with a layer of nanotubes is a difficult process, but the authors of this paper have achieved quite good experience in carrying out such procedures [1].

The implementation of the concept of using CNT coating may lead to three fundamental effects: a decrease in friction loss, an increase in surface resistance to abrasive wear, and a reduction of vibrations generated by the periodicity of piston internal combustion engine work. The basis for obtaining such effects is, however developing a method of obtaining permanent, non-exfoliating layers of nanotubes bounded to the base that is the refined surface. Next, on the nanotubes one must embed an intermediate substance of good tribological properties.

To begin with, it was assumed that platinum would be used as the intermediate substance in the experimental attempts. Due to its price, platinum certainly cannot be the target element improving the tribological properties of CNT layers. By selecting platinum at the experimental phase one can yet be sure of excluding the phenomena of reacting with reagents or oxygen contained in the air that would be difficult to control.

\section{Application of enriching substances on the layer of nanotubes embedded on metal surface}

Strength properties of CNTs, which are inaccessible for other materials, are the basic feature, which can allow for a practical elimination of abrasive wear of cooperating surfaces of kinematic pairs in a combustion engine. However, one basic condition must be fulfilled; namely, the applied layers must permanently adhere to the refined surface. Also, one must develop the procedure of applying substances of the required antifriction properties on the fibbers of nanotubes. The starting point in the performed experiments is an attempt to apply platinum particles on CNTs.

A synthesis of platinum nanoparticles enables obtaining particles of several nanometers in size, which gives the possibility of covering large areas with a small amount of costly platinum. However, it is very important for their diameter to be not less than $2 \mathrm{~nm}$. If platinum nanoparticles were smaller than $2 \mathrm{~nm}$, they will lose their properties known from the macro structures [2-11]. The platinum nanoparticles obtained as a result of the modified Turkevich method were characterized by means of the transmission electron microscope (LEO 922A). As it is shown in the pictures below Fig. 1 [1], the nanoparticles synthesized by this method are several nanometers in size.

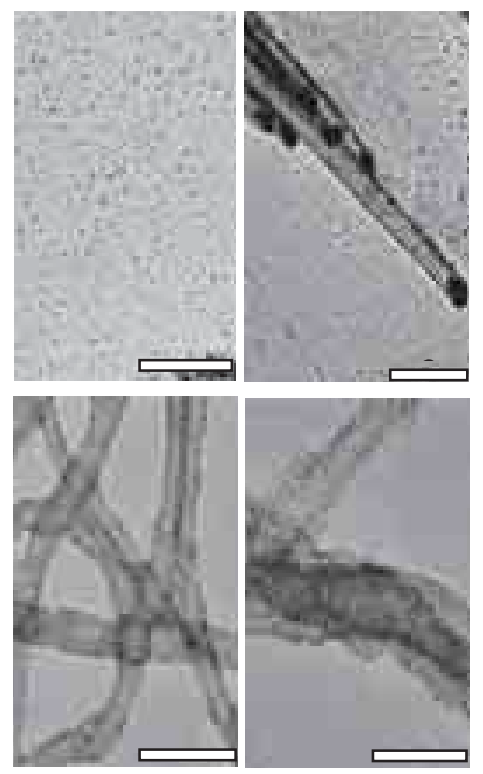

Fig. 1. Pictures from a transmission electron microscope presenting CNTs with the embedded platinum nanoparticles on them 
The difference in the modified Turkevich method is the application of a proper reducer. In the Turkevich method, the reducing element uses thermal energy obtained during heat treatment. In the modified method, one uses a stronger reducer, which is tetra sodium hydroboride, which reacts quicker, leaving smaller nanoparticles [1].

\section{Nanotube growth}

The growth in the CVD process allows for obtaining Multiwalled Carbon Nanotubes (MWCNTs) on large surfaces while achieving very good efficiency.

Conducting the growth process while using the CVD technique, at the simultaneous use of a strong electric field enabled vertical growth of nanotubes. It was possible due to large flat surfaces located in one plane. The vertical growth of nanotubes is shown in Fig. 2 A), B) and C), whereas the anisotropic growth in D).
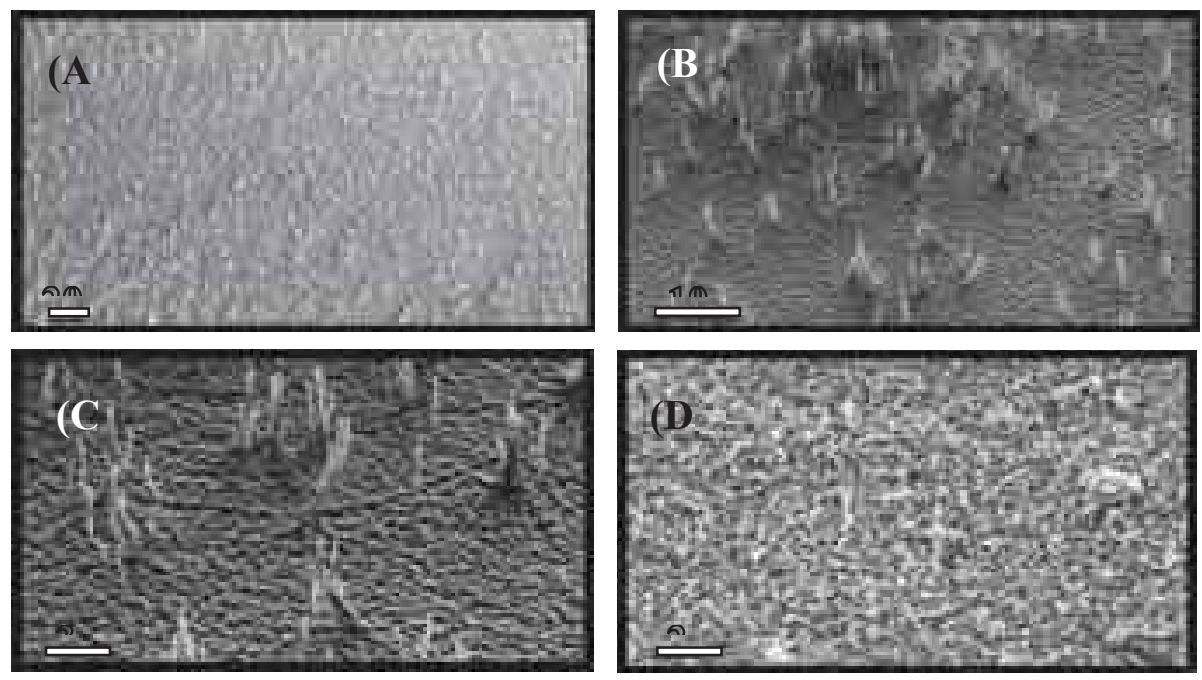

Fig. 2. Pictures from a scanning electron microscope (SEM) showing metal surface coated with CNTs

Figure 3 presents a piston selected for testing before it is coated with a layer of nanotubes, and Fig. 4 shows the same piston following the growth (on the side surface) of a layer of nanotubes which are $5 \mu \mathrm{m}$ thick.

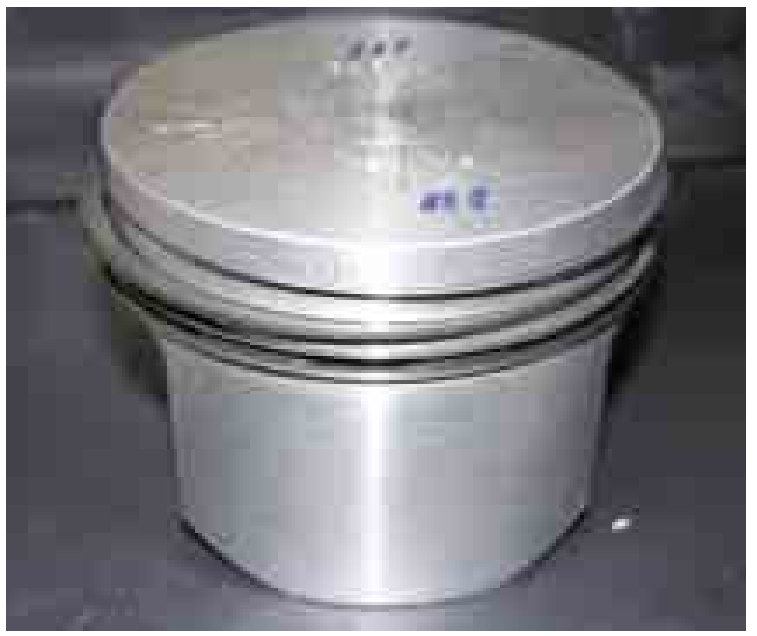

Fig. 3. The piston selected for testing before it is coated with a layer of nanotubes

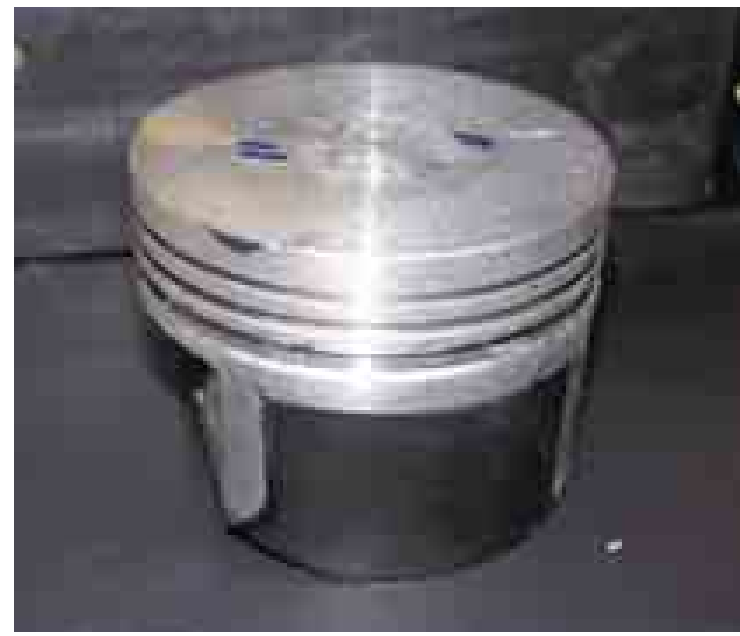

Fig. 4. The piston selected for testing when its side surface is coated with a layer of nanotubes 


\section{Test results of resistance to motion torque of a combustion engine for two variants of pistons}

The pistons presented in Fig. 3, 4 were mounted to the engine, and then comparative tests of the resistance to motion torque were carried out. Test results for a factory piston are shown in Fig. 5, and for the piston coated with a layer of CNTs - in Fig. 6-8.

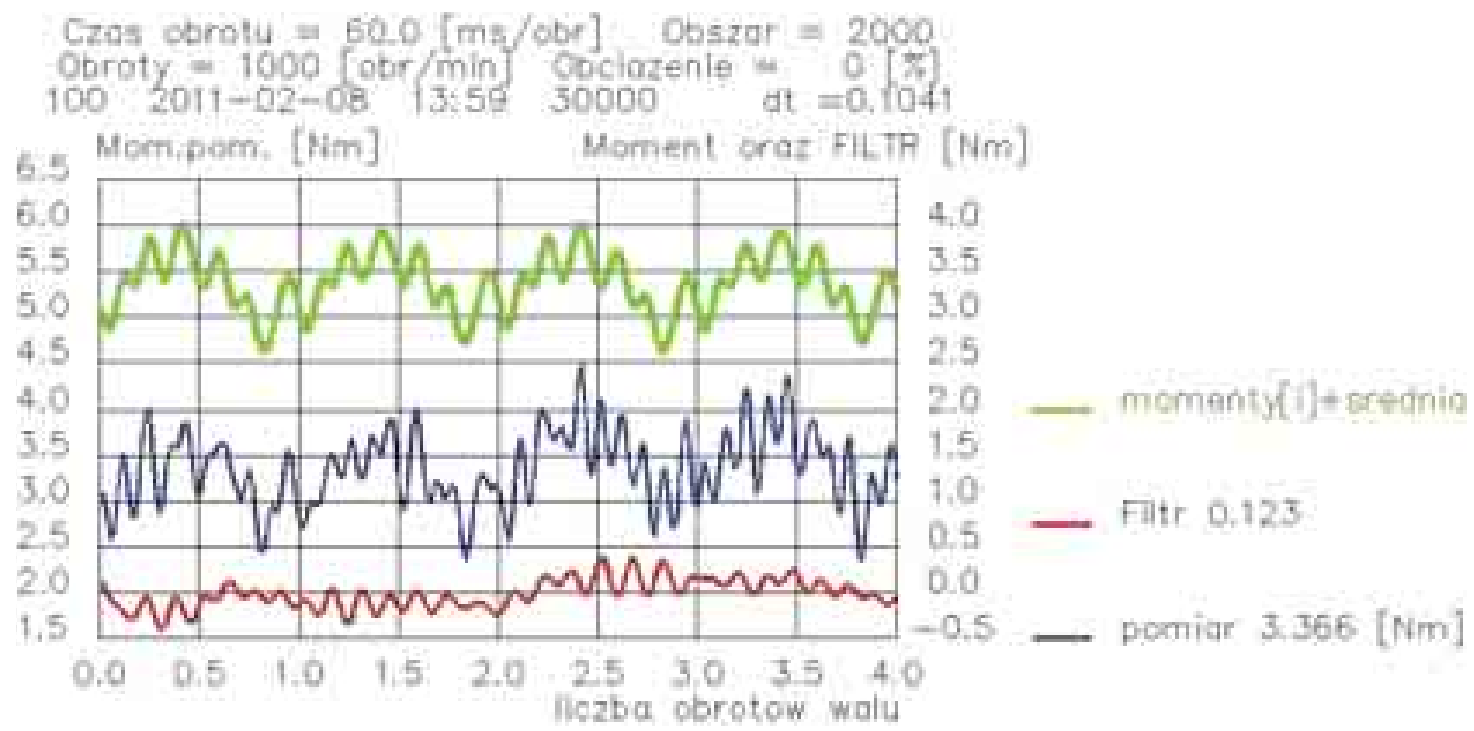

Fig. 5. The course of the engine drive torque - the blue line associated with the scale marked on the left side of the chart presents the results of a direct measurement; the course of the torque corrected by subtracting these harmonics from the measurement results which are not in the phase with the angular velocity of the enginethe green line, and a summary torque subtracted as a result of correction from the desired torque - the red line. The range of courses marked green and red is shown on the right side of the Fig. The courses obtained for standard pistons following 10 hours of engine work. Key: Czas obrotu - rotation time [ms/revolution]; Obszar - area; Obroty - revolutions; Obciażenie - load; Momenty[i]+ średnia - moments [and] + mean; Filtr-filter; Pomiar-measurement; Liczba obrotów walu-number of revolutions of the shaft

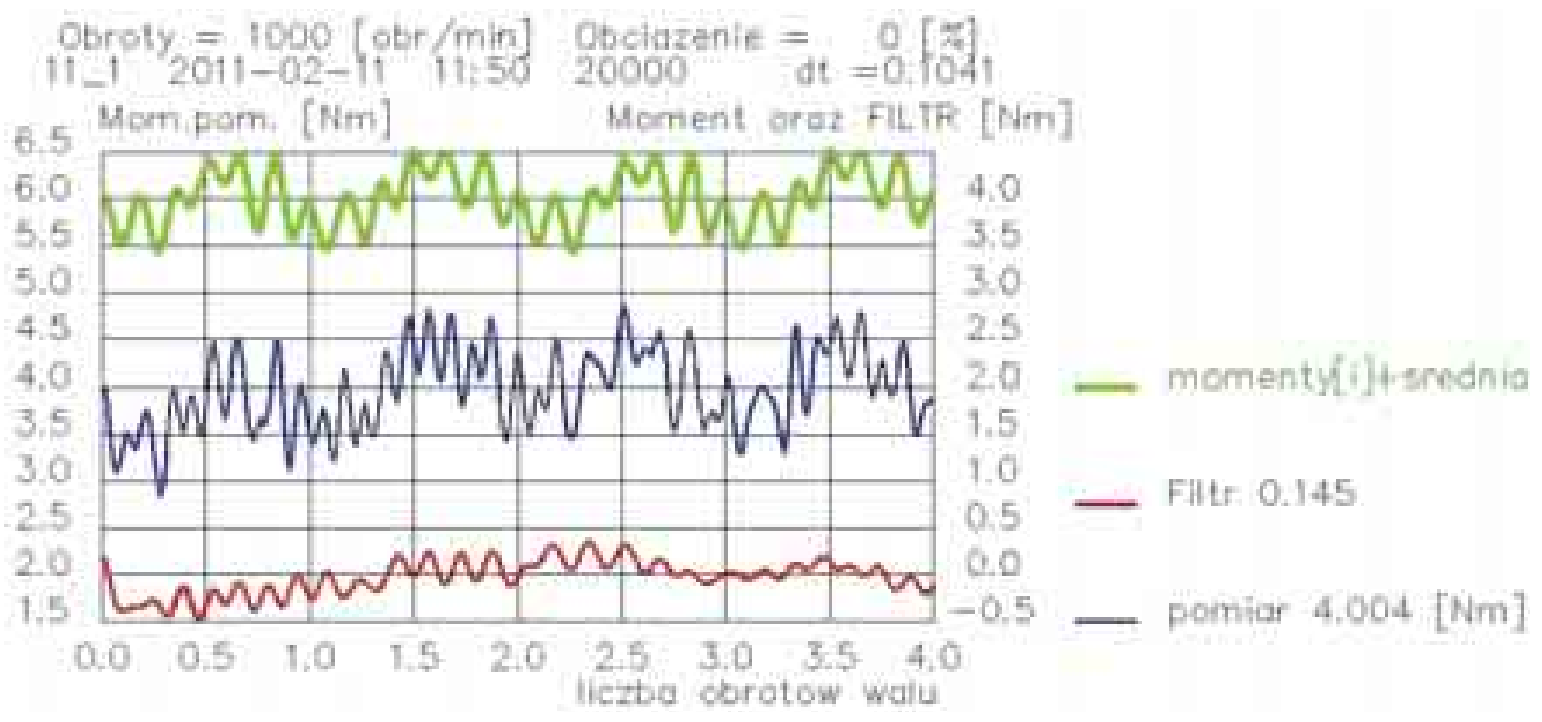

Fig. 6. The course of the engine drive torque - the blue line associated with the scale marked on the left side of the chart presents the results of a direct measurement; the course of the torque corrected by subtracting these harmonics from the measurement results which are not in the phase with the angular velocity of the enginethe green line, and a summary torque subtracted as a result of correction from the desired torque - the red line. The range of courses marked green and red is shown on the right side of the Fig. The courses obtained for standard pistons following 5 minutes of engine work 


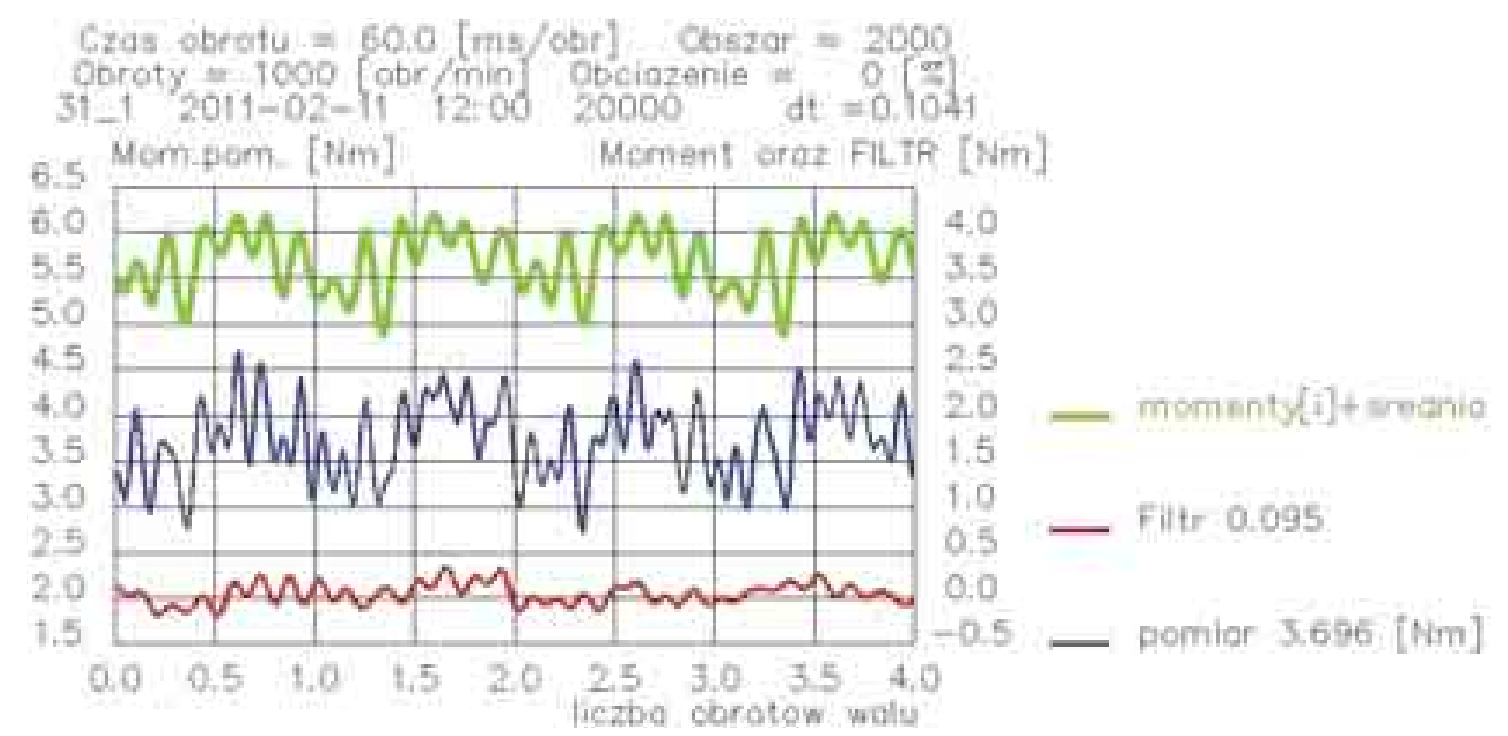

Fig. 7. The course of the engine drive torque - the blue line associated with the scale marked on the left side of the chart presents the results of a direct measurement; the course of the torque corrected by subtracting these harmonics from the measurement results which are not in the phase with the angular velocity of the enginethe green line, and a summary torque subtracted as a result of correction from the desired torque - the red line. The range of courses marked green and red is shown on the right side of the Fig. The courses obtained for standard pistons following 15 minutes of engine work

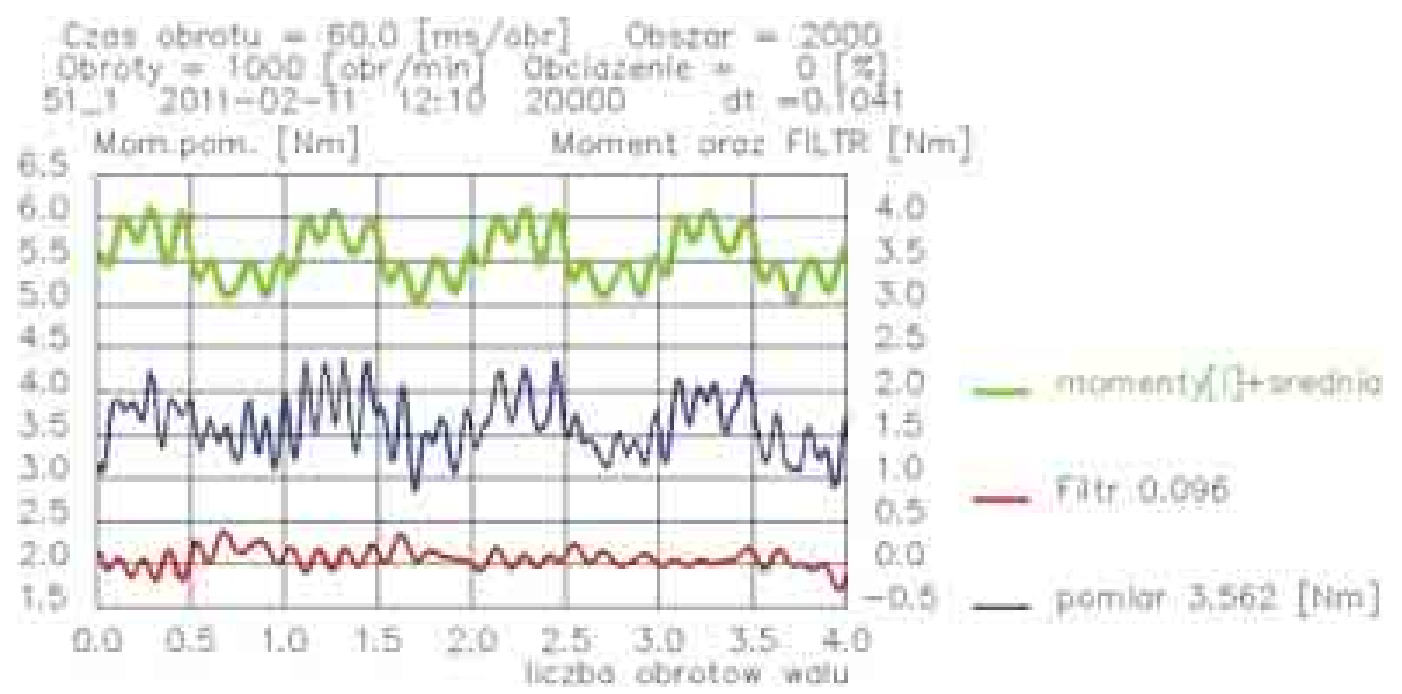

Fig. 8. The course of the engine drive torque - the blue line associated with the scale marked on the left side of the chart presents the results of a direct measurement; the course of the torque corrected by subtracting these harmonics from the measurement results which are not in the phase with the angular velocity of the enginethe green line, and a summary torque subtracted as a result of correction from the desired torque - the red line. The range of courses marked green and red is shown on the right side of the Fig. The courses obtained for standard pistons following 25 minutes of engine work

The course of torques shown in Fig. 6 was obtained after 5 minutes of engine motion, following its assembly, while the course in Fig. 7 presents the measurement results after 15 minutes of engine motion, and finally the course in Fig. 8 - after 25 minutes. The tests were performed while adopting the temperature of the cooling medium at the level of 89.5 to $90.5^{\circ} \mathrm{C}$. Fig. 9 presents the test station for performing the measurement of the coupling of the combustion engine with the electric motor that drives it.

While building the test station, special attention was paid to ensuring a stable temperature of the cooling medium, which is clearly necessary to capture the differences in internal frictional resistance generated by the tested engine. 


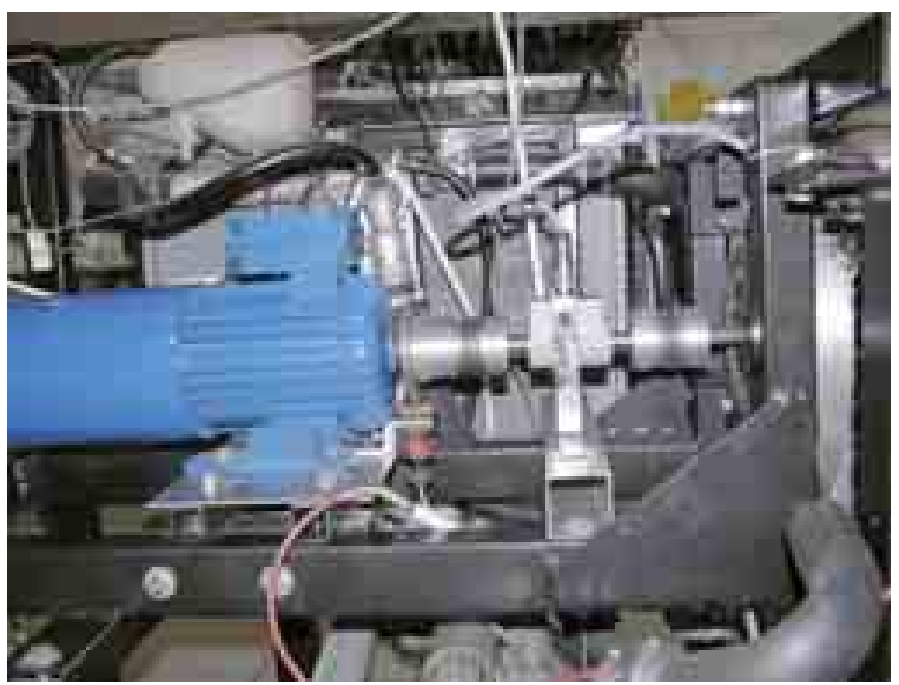

Fig. 9. General view on the test station for measuring the moment of coupling the internal combustion engine with the asynchronous electric motor that drives it

\section{Test results analysis}

By comparing the courses of torques of the combustion engine drive equipped with standard pistons and the ones coated with the layer of CNTs, one can notice the higher initial friction losses in the case of the pistons coated with CNTs, conventionally called SSNW. Following a 25-minute engine motion, the SSNW average engine moment of friction amounts to $3.56 \mathrm{Nm}$, whereas the engine equipped with standard pistons - SSTS - 3.36 Nm. In the case of the SSNW engine, one can notice however, a clear tendency to decrease the moments of resistance to motion, and besides, the clearance between the piston and the cylinder bearing surface in the SSNW engines is less than the double value of the thickness of the nanotubes, that is $10 \mu \mathrm{m}$.

The more important result of the research is the mean deviation of the torque signal of the frequency different from the multiple frequency of the rotational motion defined as the nonperiodical torque - NPT. The course of this signal is shown in Fig. 5 through 8 as red, where the values of the mean deviation are as follows:

- for the SSTS standard piston $-0.123 \mathrm{Nm}$,

- for the SSNW piston after 5 minutes of engine work $-0.145 \mathrm{Nm}$,

- for the SSNW piston after 15 minutes of engine work $-0.095 \mathrm{Nm}$,

- for the SSNW piston after 25 minutes of engine work $-0.096 \mathrm{Nm}$.

The initial value of the mean NPT deviation for the SSNW pistons amounts to $0.145 \mathrm{Nm}$, and next, it quickly decreases to the level of $0.095 \mathrm{Nm}$; while for the SSTS pistons the value of the mean NPT deviation is $0.123 \mathrm{Nm}$. This means that the SSNW pistons stabilize the motion of the piston, and this means less intense torsional vibrations and lower noise intensity.

\section{Conclusions}

1. There is a possibility of applying on the aluminum side-surface of the piston a modified layer of CNTs of very small thickness, smaller than the standard assembly clearance of the pistons mounted in the cylinder.

2. The developed layer of CNTs adheres to the aluminum surface to an extent preventing peeling of the layer due to both mechanical and thermal loads existing in the piston combustion engine driven from the outside.

3. A decrease in the clearance between the piston and the cylinder - by embedding a layer of nanotubes on the side surface of the piston - leads to a slight increase in the moment of resistance to motion, but these are the conditions limiting the torsional vibrations of the engine shaft. 


\section{Acknowledgements}

Project nr 3374/B/T02/2009/36 n.t. Redukcja niestabilności momentu generowanego przez silnik spalinowy $w$ zespołach hybrydowych is financed by Ministry of Science and Higher Education

\section{References}

[1] Ciałkowski, M, Iskra, A., Giersig, M., Kempa, K. Wysokoefektywny samochodowy reaktor katalityczny na bazie trójwymiarowych hierarchicznych nanostruktur węglowych, Nr projektu: 3940/T02/2007/32, Poznań 2009.

[2] Kinoshita, K., Electrochemical oxygen technology, John Wiley \& Sons: New York 1992.

[3] Durand, R., Faure, R., Gloaguen, F., Aberdam, D., Adzic, R. R., Ansonand, F. C., Kinoshita, K., Eds., The Electrochem. Soc. Inc., Vol. 95-26, p. 27, Pennington 1996.

[4] Kabbabi, A., Gloaguen, F., Andolfatto, F., Durand, R., J. Electroanal. Chem., 373, 251-254, 1994.

[5] Frelink, T., Visscher, W., vanVeen, J. A. R., J. Electroanal. Chem., 382, 65-72, 1995.

[6] Takasu, Y., Ohashi, N., Zhang, X. G., Murakami, Y., Minagawa, H., Sato, S., Yahikozawa, K., Electrochim. Acta, 41, 2595-2600, 1996.

[7] Cherstiouk, O. V., Simonov, P. A., Savinova, E. R., Electrochim. Acta, 48, 3851-3860, 2003.

[8] Maillard, F., Eikerling, M., Cherstiouk, O. V., Schreier, S., Savinova, E., Stimming, U., Faraday Discuss., 125, 357-377, 2004.

[9] Arenz, M., Mayrhofer, K. J. J., Stamenkovic, V., Blizanac, B. B., Tomoyuki, T., Ross, P. N., Markovic, N. M., J. Am. Chem. Soc., 127, 6819-6829, 2005.

[10] Tang, Z. C., Geng, D. S., Lu, G. X., J. Colloid Interface Sci., 287, 159-166, 2005.

[11] Mukerjee, S., McBreen, J., J. Electroanal. Chem., 448, 163-171, 1998.

[11] Sun, Y., Zhuang, L., Lu, J., Hong, X., Liu, P. J., Am. Chem. Soc., 129, 15465-15467, 2007. 